was no arterial pressure gradient between the arms and legs after the operation.

\section{Discussion}

Surgical repair of aortic coarctation has expanded to include resection with end-to-end anastomosis, ${ }^{1}$ prosthetic patch aortoplasty, ${ }^{2}$ subclavian flap aortoplasty, ${ }^{3}$ and aortic resection with graft replacement. Because further aortic growth is not a problem in adult patients, graft replacement or bypass is often used and produces the best results. ${ }^{4,5}$ As the aortic wall in the portion of coarctation was thought to be fragile in our case because of the median necrosis, we used an open proxymal anastomosis technique to avoid possible aortic injury caused by crossclamping.

The central cannulation technique ${ }^{6}$ is preferred for correction of postductal coarctation of the aorta to secure sufficient cerebral perfusion. In our case, this technique also had the advantage of preventing scattering of debris by the blood jet in an aortic aneurysm to cerebral blood flow. We prefer insertion of a venous cannula into the right atrium through the right femoral vein rather than insertion into the main pulmonary artery, as originally reported by Westaby and colleagues, ${ }^{6}$ because we have sometimes found that the wall of the main pulmonary artery is very fragile and thus susceptible to injury. We routinely insert a double-staged
}

venous cannula through the right femoral vein in operations for descending aortic aneurysms and have experienced no technical problems.

In conclusion, our "modified" central cannulation technique and open proxymal anastomosis technique seem to be safe. This is an appropriate approach for surgical correction, and we recommend it as the standard approach for the coarctation of the aorta in adults.

\section{References}

1. Crafoord C, Nylin G. Congenital coarctation of the aorta and its surgical treatment. J Thorac Cardiovasc Surg. 1945;14:347-61.

2. Vossschulte K. Surgical correction of coarctation of the aorta by an "isthmusplastic" operation. Thorax. 1961;16:338-45.

3. Waldhausen JA, Nahrwold DL. Repair of coarctation of the aorta with a subclavian flap. J Thorac Cardiovasc Surg. 1966;51:532-3.

4. Lawrie GM, DeBakey ME, Morris GC Jr, et al. Late repair of coarctation of the descending thoracic aorta in 190 patients. Results up to 30 years after operation. Arch Surg. 1981;116:1557-60.

5. Aris A, Subirana MT, Ferres P, Torner-Soler M. Repair of aortic coarctation in patients more than 50 years of age. Ann Thorac Surg. 1999;67:1376-9.

6. Westaby S, Katsumata T, Vaccari G. Arch and descending aortic aneurysms: influence of perfusion technique on neurological outcome. Eur J Cardiothorac Surg. 1999;15:180-5.

\title{
Severe pulmonary stenosis and aortopulmonary fistula caused by a dissecting aneurysm in the ascending aorta
}

\author{
Kazuhito Imanaka, MD, ${ }^{a}$ Shunei Kyo, MD, ${ }^{a}$ Haruhiko Asano, MD, ${ }^{a}$ Noboru Motomura, MD, ${ }^{c}$ Shinichi Takamoto, MD, \\ Masaaki Kato, MD, ${ }^{a}$ Masanori Ogiwara, MD, a and Osami Kohmoto, MD, Saitama and Tokyo, Japan
}

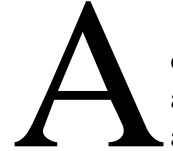

35-year-old man was referred to our hospital because of persistent fever lasting for 1 month, mild dyspnea, and continuous heart murmur. Oral and intravenous antibiotics had been administered at another hospital. The leukocyte count was $12,000 / \mu \mathrm{L}$, and the serum $\mathrm{C}$-reactive protein level was $3 \mathrm{mg} / \mathrm{dL}$. The patient had never had severe pain

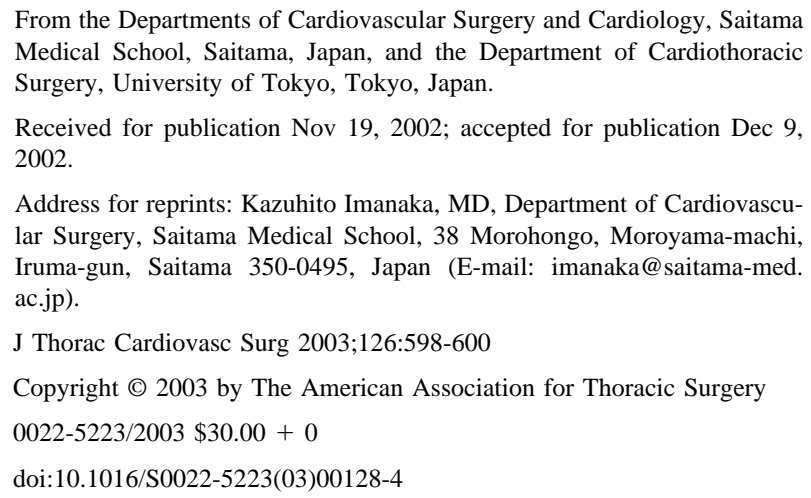

in the chest or in the back, but he had a 1-year history of multiple systemic abscesses that spontaneously developed and healed. Several imaging studies revealed a dissecting aneurysm in the ascending aorta that compressed the pulmonary artery (PA), causing severe pulmonary stenosis (pressure gradient, $75 \mathrm{~mm} \mathrm{Hg}$ on echocardiography), and that was accompanied by a fistula into the PA (Figure 1). Proximally, the aneurysm reached the level of the aortic valve annulus. A moderate amount of right pleural effusion and marked liver congestion were also noted. On that day, he was admitted to our hospital. However, severe PA stenosis precluded successful right-heart catheterization. Antibiotic therapy was discontinued, but blood culture failed to disclose causative organisms. The clinical condition of the patient remained almost stable for 5 days, but then he suddenly fell into a state of cardiogenic shock and multiple organ failure on the sixth day and underwent emergency surgical intervention. The right ventricle was severely distended and had poor contractility. Intraoperative blood sampling revealed that the $\mathrm{Qp} / \mathrm{Qs}$ ratio was 4.0. The aneurysm in front of the PA was $5 \mathrm{~cm}$ in diameter, and hematoma was absent. The almost round intimal tear in the ascending aorta, $8 \mathrm{~mm}$ in diameter, appeared to be a chronic change. The PA wall was gray and very floppy, and there was an irregularly shaped orifice of the fistula, 4 

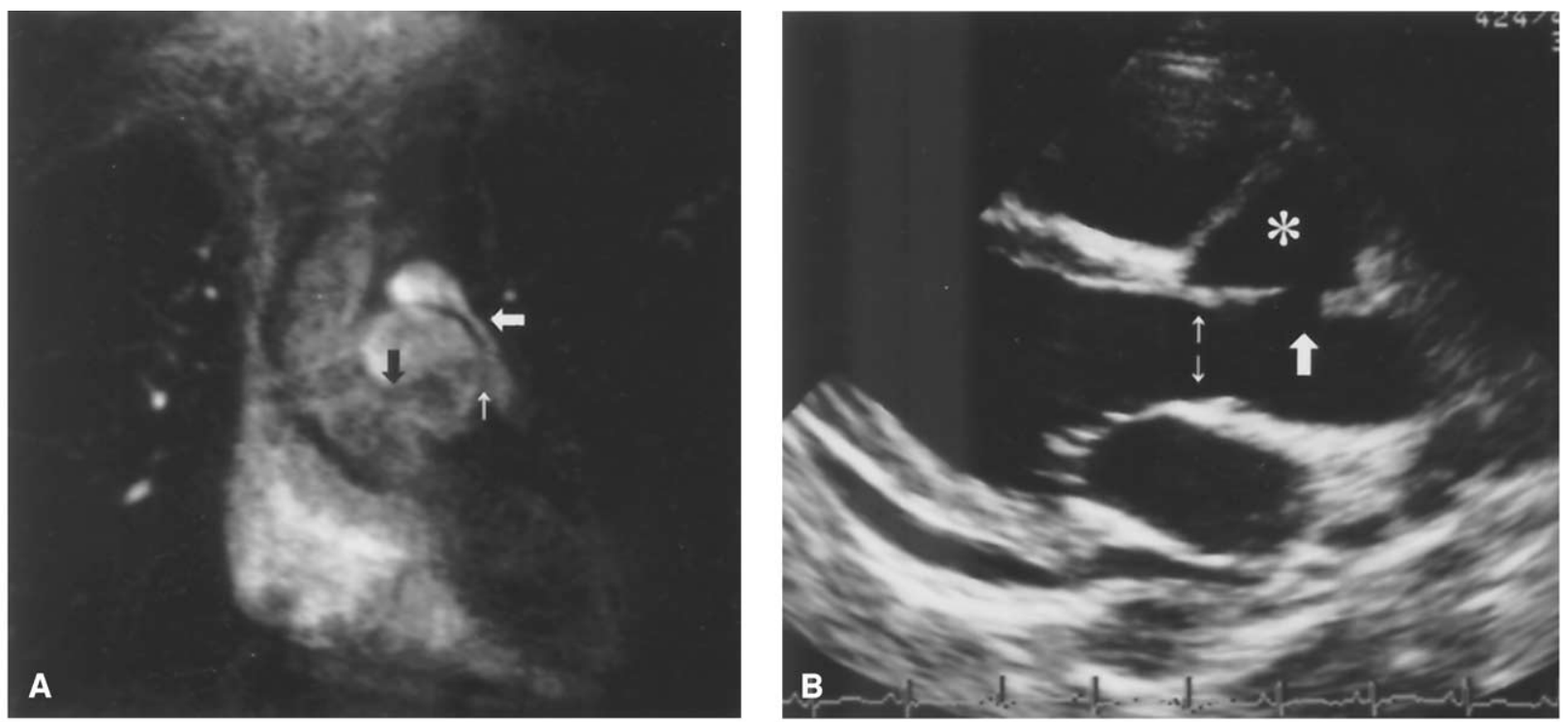

Figure 1. A, Magnetic resonance imaging (angiogram). The image shows a dissecting aneurysm in the ascending aorta (black arrow indicates the intimal tear) that compressed the PA, causing severe pulmonary stenosis (large white arrow), and that was accompanied by a fistula into the PA (small white arrow). B, Echocardiogram. Proximally, the aneurysm (asterisk) reached the level of the aortic valve annulus (small arrows). The large arrow indicates the intimal tear.

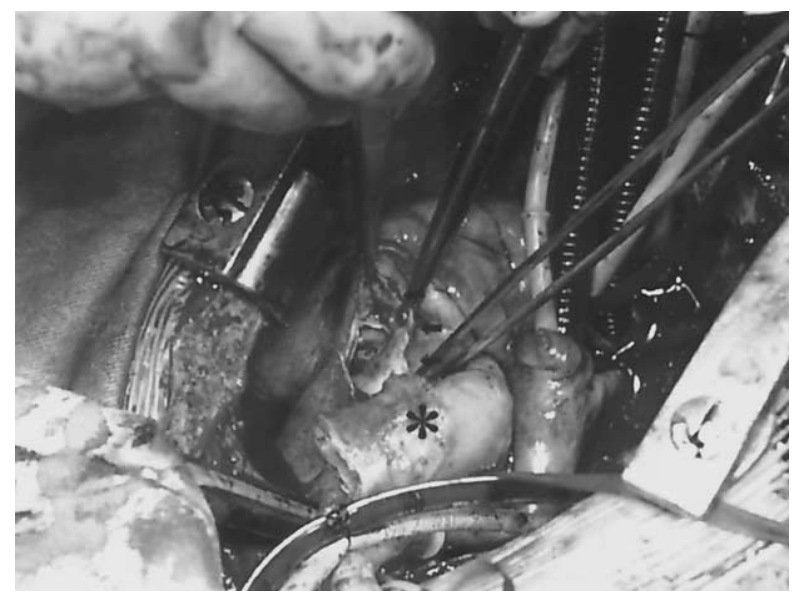

Figure 2. The PA wall (arrows) was gray and very floppy and fragile, and the PA wall around the orifice of the fistula was resected. The aortic root and the ascending aorta had already been replaced with a cryopreserved allograft (asterisk).

$\mathrm{mm}$ in size, just above the sinus of the pulmonary valve (Figure 2). The aortic root and the ascending aorta were replaced with a cryopreserved aortic allograft. The PA wall around the orifice of the fistula was resected, and the PA was reconstructed with the remnant of the aortic allograft. However, in the proximal suture line in the sinus of the pulmonary valve, the fragile PA wall was lacerated, and the resuturing had to incorporate the pulmonary valve and its annulus. The cardiopulmonary bypass was easily discontinued with a moderate amount of inotropic support.
Histologically, the resected PA wall was necrotic and had completely lost its normal structure. After the operation, residual pulmonary stenosis (pressure gradient, $40 \mathrm{~mm} \mathrm{Hg}$ ) with mild regurgitation was present and caused persistent right-heart failure. Despite good left ventricular function, central venous pressure remained greater than $15 \mathrm{~mm} \mathrm{Hg}$, and multiple organ failure did not resolve. After a nearly 4-week stay in the intensive care unit, the patient underwent PA root replacement with annulus enlargement (from $18 \mathrm{~mm}$ to $24 \mathrm{~mm}$ in diameter) using a cryopreserved pulmonary allograft. Again, he was easily separated from the cardiopulmonary bypass, but fulminant Candida species-induced mediastinitis developed, and the patient died 3 weeks later. Autopsy revealed no unacceptable structural condition in the heart.

\section{Discussion}

Aortopulmonary fistula caused by aortic dissection is a rare occurrence. Its cause has not been well documented and tends to differ from case to case. ${ }^{1-3}$ It can occur either in the acute or chronic phase of aortic dissection, either with or without previous cardiovascular surgery.

In the present case, several difficult problems were present. One was the possible close relationship between the fistula and infection. Judging from the clinical course and the laboratory data, a predisposing infection was strongly suggested. The use of prostheses should be avoided because recurrent infection is fatal. The possibly infectious aneurysm, which had reached the level of the aortic valve annulus, required extensive surgical intervention with an allograft.

Another problem was the severely damaged PA wall. Some surgeons have reported successful closure of a fistula with a 
pledget-supported suture. We did not use this method because of suspected infection and the severely damaged PA wall. The histologic findings also support our decision. However, the PA wall was extensively abnormal, and the suture line was disrupted. Resuturing incorporating the pulmonary valve annulus caused mild residual stenosis that could not be tolerated in the presence of severe right ventricular dysfunction. Pulmonary root replacement can be a method of choice in such patients. Therefore, we believe that a pulmonary allograft should also be made available whenever possible and should be implanted during the initial operation, if necessary.

The last problem was the timing of the operation. Emergency intervention was required in most reported patients with aortopulmonary fistulae and in this patient also, although the history of the present illness was rather long, and his general condition was fair at first. His severely impaired general condition 6 days later inevitably hampered postoperative recovery. Clearly, the timing of the operation should be decided on a case-by-case basis, but we recommend performing the operation as soon as possible once the diagnosis of aortopulmonary fistula is established.

\section{References}

1. Lindsay J Jr. Aortocameral fistula: a rare complication of aortic dissection. Am Heart J. 1993;126:441-3.

2. Piciche M, De Paulis R, Chiariello L. A review of aortopulmonary fistulas in aortic dissection. Ann Thorac Surg. 1999;68:1833-6.

3. Leborgne L, Trojette F, Jarry G, Touati G, Otmani A, Hermida JS, et al. Dissection of the aorta complicated by aorto-pulmonary fistula. Arch Mal Coeur Vaiss. 2001;94:743-6.

\section{Stent graft treatment for abdominal pseudoaneurysm near the celiac artery}

Shoichi Takahashi, MD, ${ }^{a}$ Shunichi Takaya, MD, ${ }^{a}$ Ikuo Fukuda, MD, ${ }^{\text {a }}$ Takemichi Suto, MD, ${ }^{a}$ Kazuyuki Daitoku, MD, ${ }^{\text {a }}$ Toshihiko Kuga, MD, ${ }^{a}$ Ikko Ichinoseki, MD, ${ }^{a}$ Mamoru Munakata, MD, ${ }^{a}$ Kozo Fukui, MD, ${ }^{a}$ Hiroshi Noda, MD, and Hiraku Yodono, MD, Aomori, Japan

$\mathrm{T}$ he patient was a 56-year-old man who had a history of abdominoperineal resection and colostomy for rectal cancer 10 years before. There was no other major medical history or trauma. He was admitted to the hospital because of fever and abdominal pain that continued for 1 month. $\mathrm{He}$ was given a diagnosis of inguinal lymphadenitis and received intravenous infusion of antibiotics. Temporarily, the fever subsided, and abdominal symptoms improved. However, a high fever soon developed, and the abdominal pain returned. The patient was transferred to our hospital. Computed tomographic scanning of the chest and abdomen revealed an aneurysm of $25 \mathrm{~mm}$ in diameter. It was located on the opposite site of the celiac axis, and there was partial calcification of the aortic wall (Figure 1). Considering the clinical context and morphology of the aneurysm, a diagnosis of mycotic aneurysm was made. Administration of antibiotics and intensive antihypertensive therapy were continued until resolution

\footnotetext{
From the Departments of Surgery ${ }^{\mathrm{a}}$ and Radiology, ${ }^{\mathrm{b}}$ Hirosaki University School of Medicine, Aomori, Japan.

Received for publication Dec 2, 2002; accepted for publication Dec 16, 2002.

Address for reprints: Shoichi Takahashi, MD, Department of Cardiac Surgery, Southern Tohoku General Hospital, 7-115 Yatsuyamada, Koriyama, Fukushima 963-8563, Japan (E-mail: s.takahashi@mt.strins.or.jp).

J Thorac Cardiovasc Surg 2003;126:600-2

Copyright $\odot 2003$ by The American Association for Thoracic Surgery $0022-5223 / 2003 \$ 30.00+0$

doi:10.1016/S0022-5223(03)00127-2
}

of fever and improvement of the inflammatory marker. The fever had subsided almost completely by 1 week after transfer. The leukocyte count was $4800 / \mathrm{mm}^{3}$, and C-reactive protein levels were negative by 6 weeks after transfer. The results of blood culture, repeated several times, were negative. Because the pseudoaneurysm had been growing rapidly (computed tomography, $35 \mathrm{~mm}$ in diameter), surgical intervention was recommended. Because of the colostomy, open surgical intervention was anticipated to have high mortality and morbidity, including graft infection. Therefore stent grafting was conducted.

The patient was operated on under general anesthesia. The right femoral artery was surgically exposed. After systemic heparinization, the femoral artery was incised, and a sheath was inserted. A handmade, half-fenestrated stent graft was prepared. We used a stainless-steel Gianturco Z stent (Cook Inc, Bloomington, Ind) 30 $\mathrm{mm}$ in diameter and $50 \mathrm{~mm}$ in length. To determine its orientation, we put radiolucent markers on 2 of the 6 apexes of the stent where the graft would cover the orifice of the pseudoaneurysm (Figure 2, $B$ ) One third of the circumference of the lower three quarters of a woven graft (Ube Inc, Ube, Japan) was cut to preserve the abdominal branches, as shown in the Figure 2, A. A fan-shaped opening was also made to preserve the thick lumbar arteries. The stent was deployed with the markers lined up when viewed from the anterior perspective to preserve the branch.

The angiogram performed after deployment confirmed that the aneurysm had been excluded and that the abdominal branches were patent.

The patient was discharged without any serious complication. No signs of infection were observed for longer than 10 months after the operation. 